\title{
ALPHA CHANNELING IN MIRROR MACHINES AND IN TOKAMAKS
}

\author{
Nathaniel J. Fisch \\ Department of Astrophysical Sciences, Princeton University, Princeton, NJ 08543 \\ fisch@princeton.edu
}

The alpha channeling effect in tokamaks exploits the higher population of high-energy alpha particles in the tokamak interior compared to that of low-energy alpha particles at the periphery. Because of the population inversion, it is possible to inject waves that diffuse resonant particles along diffusion paths connecting these regions, so that hot alpha particles diffuse to the periphery and cool at the same time. This effect has been suggested as a way of achieving considerably higher performance in tokamak fusion reactors, and similar possibilities might be expected in mirror reactors.

\section{Introduction}

In tokamaks, operation in the hot ion mode, where ion temperatures exceed electron temperatures, gives higher effective fusion reactivity. ${ }^{1}$ However, in fusionignited plasma, the electron temperature exceeds the ion temperature, because the energetic alpha particles preferentially heat electrons. For ions to be hotter than electrons, what is required is alpha channeling, an effect that taps the free energy in the alpha particles on a collisionless time scale so that this energy is given up to waves, which might then damp directly on ions, and at the same time expelling the fusion ash. ${ }^{2}$ Channeling $75 \%$ of the alpha particle energy to ions can result in more than a factor of two in reactivity. ${ }^{3}$ The channeled wave energy might also be used for other useful purposes like current drive. ${ }^{4}$ These effects all lead to a much more economical tokamak reactor. $^{5}$

Similar advantages in channeling alpha particle energy on a collisionless time scale might be expected in mirror machines. The open geometry of mirror machines makes fueling and ash removal much easier, but removing quickly the spent fuel in mirror devices does free up valuable electric potential for fuel ions. However, mirror and other open field line reactor concepts tend to suffer from very high auxiliary power requirements, ${ }^{6-7}$ so employing the channeled energy so as to decrease the circulating power may be the most critical gain.

To see how the channeling might happen, suppose a wave diffusion path in the joint velocity-configuration space that connects hot particles in the center of a plasma device with cold particles on the periphery of the device.
Particles diffused by waves must then necessarily leave the device cold, giving up their energy to the waves that produce the particle diffusion. ${ }^{2}$

This population inversion occurs naturally in a DT fusion reactor, where energetic $3.5 \mathrm{MeV}$ alpha particles are produced in the reactor interior, mostly at the reactor core, where the fuel constituents are hottest and densest. However, near the periphery of the device, very few alpha particles are produced, and those that might be found near the periphery have generally slowed down to the much lower fuel temperature. The alpha particle distribution thus has the appropriate population inversion. If appropriate waves are employed, alpha particles must give up energy to waves on a collisionless time scale, while simultaneously exiting the reactor as cold ash.

Note that there are stringent conditions on the particle diffusion. Fig. 1 shows schematically an alpha particle distribution function $f_{\alpha}$ as a function of energy $\varepsilon$ both near the tokamak axis at $r=0$ and near the tokamak periphery at $r=a$. Here, even if the distribution function at the tokamak center were monotonically decreasing in energy, a diffusion path that connects high-energy center particles with low-energy peripheral particles nonetheless exploits population inversion and extracts energy. Particles can diffuse along the diffusion path either to higher energy or to lower energy, but only at lower energy do the particles encounter the periphery and exit. Thus, absent collisions, eventually, all particles must leave at low energy.

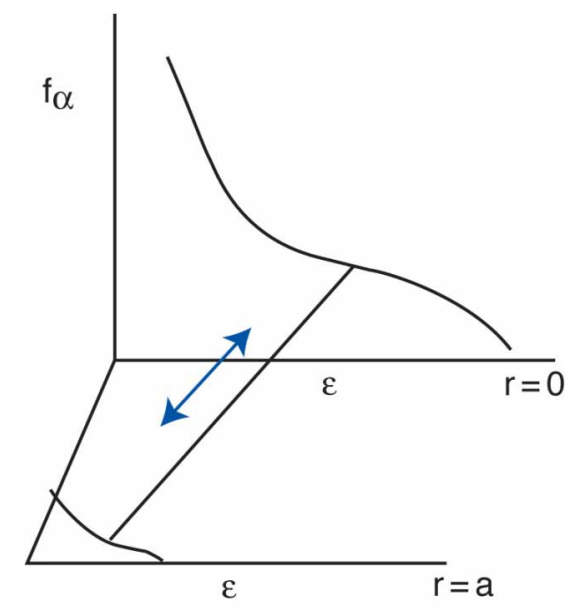

Fig. 1. Diffusion path from hot core to cold periphery. 
Note as well that in a tokamak the population inversion for fuel deuterium ions is opposite to that of the alpha particles. There are no $\mathrm{MeV}$ fuel ions in the center, but there are many relatively cold fuel ions near the periphery. Thus, in a tokamak, the same diffusion path that allows waves to tap alpha particle energy, while rejecting the alpha particles to the periphery, might also allow waves to fuel the plasma by sucking in fresh fuel ions and heating them. The quick rejection of alpha particles and the channeling of alpha particle energy to cold ions, which are then drawn to the plasma core, would all be extremely useful effects in tokamaks.

We first discuss the channeling effect in a tokamak, where it is more highly developed. For mirror machines the periphery and the core for confined alpha particles are defined very differently than for tokamaks.

\section{The Alpha Channeling Effect}

To see how diffusion paths can be arranged in joint velocity-configuration space to produce cooling ${ }^{2}$, consider for simplicity slab geometry, with ions immersed in a $z$ directed magnetic field, following circular paths in the $x-y$ plane. Suppose that due to a resonant wave interaction at $v_{x}=\omega / k_{x}$, the velocity in the $x$-direction changes instantaneously as $v_{x} \rightarrow v_{x}+\Delta v_{x}$. The change in the guiding center is then $y_{g c} \rightarrow y_{g c}+\Delta v / \Omega$, where $\Omega$ is the gyrofrequency. Thus, in Fig. 2, an ion in the middle orbit undergoes a velocity kick $\Delta v_{x}$ at either point where the three orbits intersect. If the ion gains energy from the kick, it goes into the top orbit, whereas if it loses energy it goes into the bottom orbit. Thus, the change in energy $\Delta \varepsilon$ is linked to the change in the gyrocenter, with $\Delta y_{g c}$ $=\Delta \varepsilon k_{x} / \Omega m \omega$. Thus, particles that diffuse to higher energy must go up; particles that diffuse to lower energy must go down.

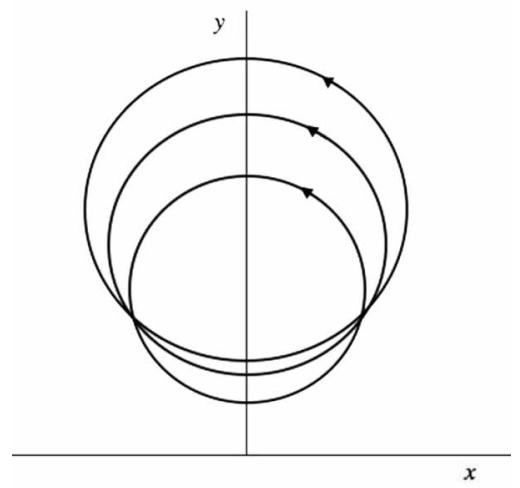

Fig. 2. Coupling of kick in energy and gyrocenter

The cooling effect works when two conditions are satisfied: First, the diffusion path must connect hot particles at the core to cold particles at the periphery. Second, the cold particles must be removed at the periphery, while the particle diffusion to higher energy must be bounded. For example, Fig. 3 depicts how electrostatic waves with phase velocity in the $y$-direction diffuse alpha particles in energy and in the $x$-direction. Alpha particles born with $3.5 \mathrm{MeV}$ near the center at $x=0$ must exhaust cold at lower energy at the periphery $x=a$. Here, the diffusion to higher energy is bounded since particles have limited excursion length to the center. The line $E=E_{\text {min }}$ represents the minimum particle energy for resonant interaction with the wave. Note that the diffusion occurs along highly constrained orbits; absent other effects such as collisions, ions must exhaust cold because there is no other way to leave the magnetic trap.

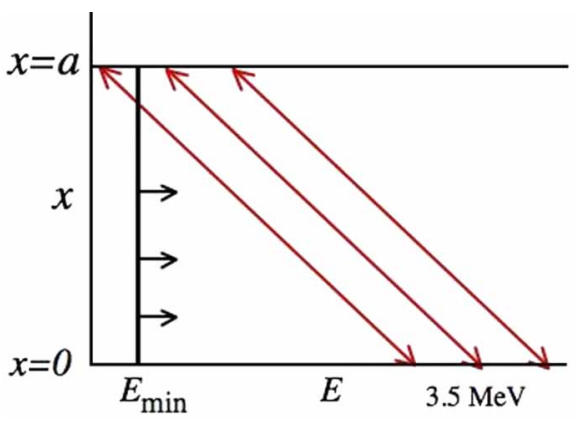

Fig. 3. Diffusion of alpha particles to periphery $\mathrm{x}=\mathrm{a}$.

Note that were the wave phase velocity reversed, the slope $d x / d E$ becomes positive, so then ions would be heated as they leave the trap. The reason for this can be seen from Fig. 4. The wave traveling to the right interacts with the particle on the bottom of its orbit, where the particle also goes to the right, while the wave going to the left interacts on the top of the orbit. Since it is important to exhaust cold rather than hot, it is critical to choose waves with the proper phase velocity.

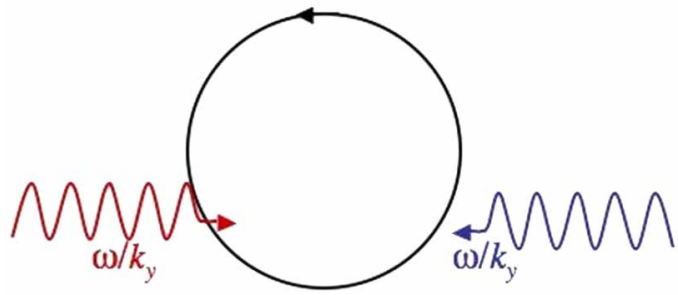

Fig. 4. Wave-particle resonance up-down asymmetry.

\section{Implementing Alpha Channeling in Tokamaks}

The alpha channeling effect in a tokamak occurs in toroidal geometry, rather than slab geometry, but the basic idea remains that diffusion paths need to connect high energy at the core with low energy at the periphery. The mode-converted ion Bernstein wave has certain appropriate wave characteristics, ${ }^{8}$ in part because upon mode conversion the high mode numbers or low phase velocities necessary for alpha channeling are generated. ${ }^{9}$ 
Schematically, as shown in Fig. 5, the mode-converted ion Bernstein wave enters the tokamak poloidal crosssection with an up-down asymmetry; the high perpendicular wavenumber in the horizontal direction then results in an asymmetric poloidal wave spectrum. More precisely, the resonant condition results in an asymmetric toroidal resonant wavenumber as well. As depicted in Fig. 5, particles then either gain energy and pinch inwards in minor radius, or lose energy and are ejected outward in minor radius.

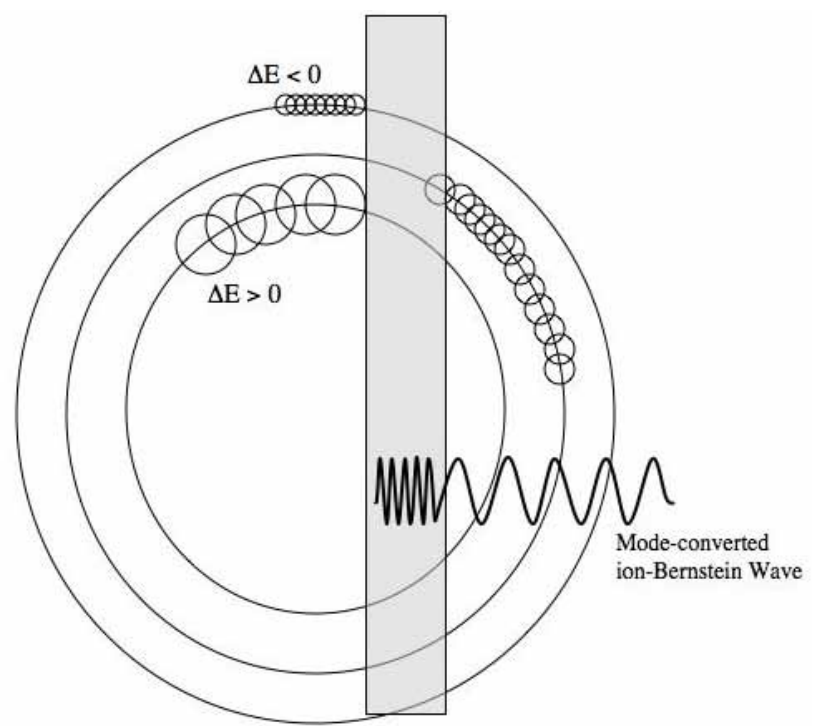

Fig. 5. Poloidal cross-section of a tokamak. The shaded region shows the mode-converted IBW wave with a short wavelength in DT plasma. The deuterium resonance is to the right (low field side) of the shaded region; the tritium resonance is to the left.

With one wave with the proper phase velocity, the constraints on the particle motion are strict; particles must exhaust cold. However, it may not be possible to excite the wave with the precisely required wave characteristics, and were it possible, it might be that not all alpha particles could resonate with it. Greater flexibility, however, can be gained by using more than one wave. Although the firm constraints on the diffusive process are relaxed, to the extent that that it is no longer enforced that particles exhaust cold, the predominant tendency can still be that particles do exhaust cold. ${ }^{10}$ Using both the mode converted IBW and a lower frequency wave in proposed reactor designs, it can be predicted that more than $2 / 3$ of the alpha particle energy might be extracted. ${ }^{11}$

\section{Experimental Results on TFTR}

Experiments on TFTR in the 1990's demonstrated that mode-converted Ion Bernstein waves could be excited in a DH plasma, and that a deuterium beam could be heated and ejected by mode-converted IBW waves. ${ }^{12-13}$ The ions exiting the tokamak could be diagnosed in detail. ${ }^{14}$ The particles were detected as a function of poloidal angle, pitch angle, energy and time, leaving no doubt as to the responsible wave-particle diffusion process. It was thus manifestly clear that the deuterium ions were diffused along diffusion paths that connected $80 \mathrm{keV}$ deuterium ions in the center of TFTR with $2.3 \mathrm{MeV}$ deuterium ions at the periphery, where they were expelled and detected. It is noteworthy that in these experiments the effective diffusion coefficient was about a factor of 50 larger than what would be predicted from quasilinear and wave tracing theory. ${ }^{15-17}$ Explanations of anomalously higher, rather tan lower, diffusion rates are hard to come by. A possible explanation, and so far the only plausible one, was that a lightly damped internal mode was ringing with the external excitation. ${ }^{15-18}$ Although this hypothesis was not verified in TFTR prior to its shutdown, the enormous advantage of a ringing internal mode is that it could make the injected energy requirement in a reactor much smaller.

\section{Implementing Alpha Channeling in Mirrors}

Both mirrors and tokamaks are devices with a symmetry direction, so that highly constrained diffusion paths can be written similarly. However, in mirror machines particles can leave not only radially across magnetic field lines, but also axially along open field lines through a loss cone in velocity space.

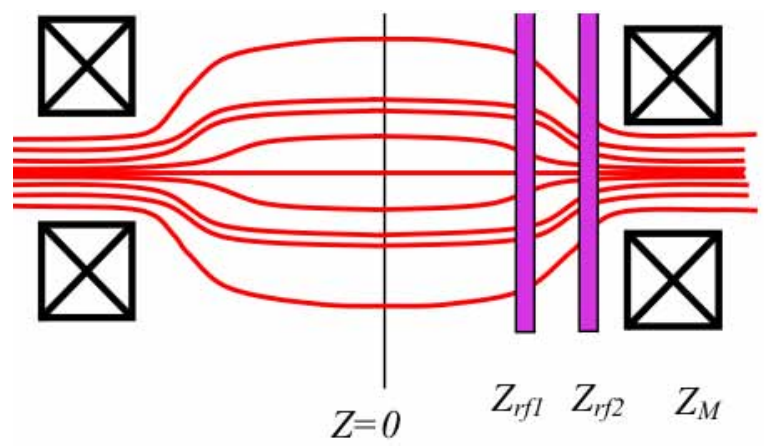

Fig. 6. RF Interaction Regions

To illustrate how the channeling effect might be constructed for a mirror machine or for that matter any of the variations on open field line magnetic traps, consider for simplicity and for didactic purposes the simple mirror, held at ground potential. Trapped alpha particles bounce between turning points with axial location $Z<Z_{M}$, where $Z_{M}$ is the axial location of the magnetic field maximum, at which point the mirror ratio $R=B(Z) / B(Z=0)$ can be defined as $R_{M}$. Suppose further that multiple regions of $\mathrm{rf}$ waves interact resonantly with alpha particles, with region $i$ occupying an axial location $Z_{r f i}$ (see Fig. 6), at which point the local mirror ration is $R_{r f i}$. Each region of radio 
frequency waves has its own frequency and wavenumber. Particles resonant in one region of $\mathrm{rf}$ waves need not be resonant in another region of rf waves, even though they may pass through that second region. Although the simple mirror machine does not make a viable fusion reactor, it can illustrate the possibilities in alpha channeling. Accordingly, following Ref. 19, what we set out to do is to demonstrate that the frequencies and wavenumbers in each rf region can be chosen such that essentially all alpha particles exhaust cold from the mirror, while yielding their birth energy to the waves. Particles trapped in the simple mirror have perpendicular and parallel energies, $W_{\perp}(Z)$ and $W_{\|}(Z)$. It is convenient, however, to refer to their perpendicular and parallel energies as they cross the mirror midplane at $Z=0$, with $W_{\perp 0}=W_{\perp}(Z=0)$, and $W_{\| 0}=W_{\|}(Z=0)$.

Suppose now that particles going through a region of rf waves at $Z=Z_{r f i}$ interact resonantly with the waves, receiving kicks in energy such that $W_{\perp}(Z) \rightarrow W_{\perp}(Z)+$ $\Delta W_{\perp}$ and $W_{\|}(Z) \rightarrow W_{\|}(Z)+\Delta W_{\|}$. For example, consider a wave-particle interaction through the resonance $\omega-k_{\|} \mathcal{V}_{\|}$ $=\Omega$, where $\Omega=q B / m$ is the local alpha particle cyclotron frequency, $\omega$ is the wave frequency, $k_{\|}$is the wave parallel wavenumber, and $v_{\|}$is the particle parallel velocity. For this resonance condition, the kicks in energy are then related by $\Delta W_{\perp}=\Delta W_{\|}\left(\Omega / k_{\|} v_{\|}\right)$. Thus, in the limit that $k_{\|} v_{\|} \rightarrow 0$, we also have $\Delta W_{\|} \rightarrow 0$, so that the kicks are in the perpendicular direction only. Since the resonance condition depends only on the parallel energy, in the limit that the energy kick is in the perpendicular direction only, particles continuing to traverse the region of $\mathrm{rf}$ remain in resonance with the wave.

Since the wave-particle interaction is a stochastic process, the energy kicks, $\Delta W_{\perp}$ and $\Delta W_{\|}$, can be positive or negative, resulting in energy diffusion. In the midplane coordinates, energy kicks $\Delta W_{\perp}$ and $\Delta W_{\|}$at $Z=Z_{r f}$ then result in midplane energy changes

$$
\begin{aligned}
& W_{\perp 0} \rightarrow W_{\perp 0}+\Delta W_{\perp} / R_{r f}, \\
& W_{\| 0} \rightarrow W_{\| 0}+\Delta W_{\|}+\Delta W_{\perp}\left(1-R_{r f}^{-1}\right),
\end{aligned}
$$

with the consistent radial coordinate change

$$
r_{0} \rightarrow r_{0}+R_{r f}^{1 / 2} \Delta W_{\perp} k_{\vartheta} / q B \omega
$$

where $r_{0}$ is the radial coordinate at the midplane and $k_{\theta}$ is the azimuthal wavenumber. Note that the diffusion in energy and radius are thus linked, with the appropriate sign of the azimuthal wavenumber linking increase in energy to decrease in radius.

To construct the channeling effect, note from Eq. (1) and Eq. (2) that for the case of perpendicular diffusion only, i.e. for $\Delta W_{\|}=0$, that the slope of the energy change $W_{\perp 0} / W_{\| 0}=\left(R_{r f}-1\right)^{-1}$, which is the same slope as the trapped-passing boundary for particles mirroring exactly at the location of the rf interaction. This means that in midplane coordinates the rf waves diffuse particles parallel to the associated trapped-passing boundary, with particles remaining in resonance throughout the diffusion process. The resonance condition gives the parallel energy of particles capable of exchanging energy with the wave, so the particles resonant in the rf region at $Z=Z_{r f}$ have parallel energy $W_{\| \text {res }}$ whereas particles mirroring at $Z=Z_{r f}$ by definition have vanishing parallel energy. Thus, the diffusion path is offset from the trapped-passing boundary by exactly $W_{\| \text {res }}$. In Fig. 7 , the energy diffusion paths in midplane coordinates are schematically presented for particles resonating with the two rf field regions depicted in Fig. 6. The solid rays are the trapped-passing boundary for particles mirroring at the $\operatorname{rf}$ region $Z=Z_{r f l}$ (upper ray) and at $Z=Z_{r f 2}$ (lower ray). Each dashed line is parallel to the corresponding ray and is offset from the corresponding trapped-passing boundary by the corresponding resonant parallel energy.

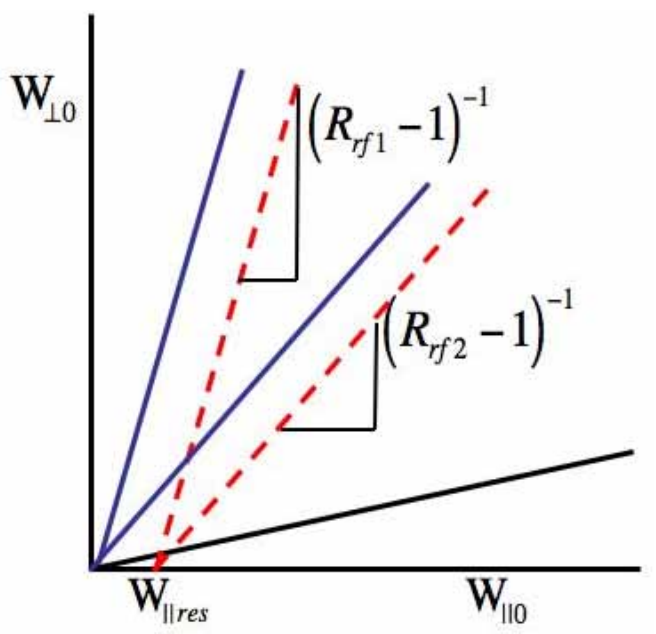

Fig. 7. Energy diffusion paths in midplane coordinates for particles resonating with the rf fields shown in Fig. 6. The upper solid rays are the trappedpassing boundary associated with each rf region and the dashed rays are the diffusion paths for particles that are resonant with the corresponding rf region. The lowest solid ray is the trapped-passing boundary associated with the maximum magnetic field.

The resonant parallel energy in each rf region is a function of local wave parameters and magnetic field strength. Fig. 7 depicts how, by choosing the same resonant parallel energy $W_{\| \text {res }}$ for particles resonating at different axial locations, all the resonant diffusion paths can meet at a point outside the trapped-passing boundary. Because these paths do not intersect within the trapped 
region of energy space, particles are resonant within only one region of $\mathrm{rf}$ power, even though they may traverse many regions of $\mathrm{rf}$ power. Thus, diffusion along each diffusion path is by construction made independent of the diffusion along all other paths, thereby constraining the diffusion to remain on one path. Clearly, by construction, the rays that meet at a point do fan out to cover essentially the whole trapped alpha particle distribution. Moreover, by choosing $W_{\| \text {res }}$ small, the diffusing resonant alpha particles necessarily encounter the trapped-passing boundary at low energy.

Since the only collisionless route out of the magnetic mirror is at low energy, alpha particles must all exhaust cold, having yielded essentially all their energy to the wave field, provided that the excursion to high energy is bounded. Although in principle the bound to high energy can occur naturally if the diffusion coefficient at high energy is small, ${ }^{20}$ the particles could leak energy if the small diffusion coefficient makes the energetic particle population susceptible to slowing down collisions. A more efficient means is to block the route to high energy altogether by tying the diffusion in energy to diffusion in radius, using one sign of poloidal wavenumber, with the radial diffusion given by Eq. (3). Thus, the alpha particles can be forced to larger radius when they diffuse to lower energy, but to smaller radius when gaining energy. The excursion to high energy is then limited, as in the case of the tokamak, by the limited radial excursion possible to lower radius. However, in contrast to the case of the tokamak, the particles could exhaust cold either through radial diffusion to the radial periphery or to he axial periphery through the velocity space diffusion to the loss cone.

\section{USES OF AMPLIFIED RF WAVES}

In tokamaks, the main uses of diverting the alpha particle energy to plasma waves would be to sustain the hot ion mode or to drive toroidal current. There is a long history in using rf waves in mirror machines. Many different $\mathrm{rf}$ waves have been used to heat mirror machines, but also there has been a worry of rf pump-out of fuel ions. ${ }^{21-22}$ However, rf waves have also be used to trap fuel ions, ${ }^{23-24}$ where the trapping occurs by heating ions through the loss cone. Here there is a fueling effect too, but it is a fundamentally different effect, since it relies upon diffusion paths in the joint energyconfiguration space. In both tokamaks and mirrors, the diffusion paths, along which alpha particles are diffused to the periphery while being cooled, can in principle also be used for ion fueling, since the population inversion is inverted for fuel ions if the ion fuel is dense and cold on the periphery, however that periphery is defined.

Like for the tokamak, there could be large advantages in maintaining different temperature ion populations. But there might also be interesting new uses for power diverted to $\mathrm{rf}$ waves such as creating transport barriers ${ }^{25}$ or maintaining sloshing ion distributions. ${ }^{26}$ Like in the case of tokamaks, all of the uses of waves might be enhanced considerably if the injected wave power were coupled to a lightly damped internal mode in the ion cyclotron frequency range. ${ }^{27-29}$ Ringing an internal mode can lead to much faster cooling of the alpha particles with less injected power. Like in the case of tokamaks, there is also an immediate use in ejecting the alpha ash quickly, since in both cases the space occupied by the alpha ash is better taken up by fuel ions.

\section{CONCLUSIONS}

In summary, although the specific implementations remain highly speculative, alpha particles born either in tokamak fusion reactors or in mirror fusion reactors might in principle be made to slow down collisionlessly on $\mathrm{rf}$ fields rather than on electrons. The alpha particle power would then be transferred or channeled to the rf fields rather than to the electrons, with the cold alpha particles promptly expelled. The quick expulsion of the spent fuel and the use of the channeled alpha particle power for purposes other than mere electron heating could then have implications for the economic viability of fusion power through the magnetic confinement of hot plasma.

In tokamaks, the energy channeled to the rf fields might be useful for driving currents or maintaining a hotion mode. In an open magnetic field system, there are other uses for the alpha particle power. However, the simple mirror considered here is by itself not a viable reactor concept. In the simple mirror, there would only be limited benefit through the channeling effect, since there is little free alpha particle energy to be channeled. More likely, the effects proposed here would be useful in an open field geometry that is closer to a reactor, such as the tandem mirror. To the extent that significant alpha particle power is produced, the alpha channeling effect could then optimize the use of this power.

Although it remains to make concrete proposals for specific reactor enhancements, it stands to reason that in both tokamaks and mirrors the diversion of alpha particle power could make a very significant change to how reactors might operate. With the channeled alpha particle power available for other purposes, the reactor could operate far from thermal equilibrium. However, at present, these tantalizing prospects must be considered highly speculative.

Nonetheless, what has been demonstrated is that, both in tokamaks and in mirror machines, a set of waves in principle exists which can produce the interesting collisionless effect that alpha particles born on the inside of the fusion device must leave the device cold, with the alpha energy transferred to the set of waves, and with the alpha cooling effect occurring concomitantly with the ejection of the alpha particles. And in both tokamaks and 
mirror machines, the vast preponderance of the alpha particles can be thus affected.

\section{ACKNOWLEDGMENTS}

This work supported by the US DOE under Grant No. DE-FG02-06ER54851.

\section{REFERENCES}

[1] J. F. CLARKE, "Hot-Ion-Mode Ignition in a Tokamak Reactor," Nucl. Fusion 20, 563 (1980).

[2] N. J. FISCH and J. M. RAX, "Interaction of Alpha-Particles with Intense Lower Hybrid Waves," Physical Review Letters 69, 612 (1992).

[3] N. J. FISCH and M. C. HERRMANN, "Utility of Extracting Power from Alpha Particles by Waves," Nuclear Fusion 34, 1541 (1994).

[4] N. J. FISCH, "Theory of RF Current-Drive," Reviews Modern Phys. 59, 175 (1987).

[5] G. A. EMMERT et al., "Improvement in Fusion Reactor Performance due to Ion Channeling," Fusion Tech. 26, 1158 (1994).

[6] R. F. POST, "Critical Conditions for SelfSustaining Reactions in the Mirror Machine," Nucl. Fus. Suppl. 1, 99 (1962).

[7] T. K. FOWLER and M. RANKIN, "Fusion Energy Balance in Mirror Machines," J. Nucl. Energy C 8, 121 (1966).

[8] N. J. FISCH et al., "Alpha Channeling Using Ion Bernstein Waves," Phys. Plasmas 2, 2375 (1995).

[9] E.J. VALEO and N.J. FISCH, "Excitation of Large- $k_{\theta}$ Ion Bernstein Waves in Tokamaks," Phys. Rev. Lett. 73, 3238 (1994).

[10] N. J. FISCH and M.C. HERRMANN, "Alpha Power Channeling with Two Waves," Nuclear Fusion 35, 1753 (1995).

[11] M. C. HERRMANN and N. J. FISCH, "Cooling a Birth Distribution of $\alpha$-particles in a Tokamak with Waves," Phys. Rev. Lett. 79, 1495 (1997).

[12] D. S. DARROW et al., "Alpha Particle Losses from Tokamak Fusion Test Reactor DT Plasmas," Phys. Plasmas 3, 1875 (1996).

[13] D. S. DARROW et al., "Enhanced Loss of Fast Ions During Mode Conversion Ion Bernstein Wave Heating in TFTR," Nucl. Fusion 35, 509 (1996)

[14] S. J. ZWEBEN et al., "Alpha Particle Physics in the Tokamak Fusion Test Reactor Experiment," Plasma Phys. and Controlled Nuclear Fusion Research 39, A275 (1997).

[15] N. J. FISCH and M. C. HERRMANN, "A Tutorial on Alpha Channeling," Plasma Physics and Controlled Fusion 41, A221 (1999).
[16] N. J. FISCH, "Physics of $\alpha$-Channeling and Related TFTR Experiments," Nucl. Fusion 40, 1095 (2000).

[17] M. C. HERRMANN, "Cooling $\alpha$-particles with Waves," Ph.D. Thesis, Princeton Univ. (1998).

[18] D. C. CLARK and N. J. FISCH, "Fast Ion Diffusion by Driven Contained Modes," Phys. Plasmas 7, 2923 (2000).

[19] N. J. FISCH, "Alpha Channeling in Mirror Machines," Phys. Rev. Lett. 97, 225001 (2006).

[20] C. F. F. KARNEY, Stochastic Ion Heating by a Lower Hybrid Wave 2, Phys. Fluids 22, 2188 (1979)

[21] D. E. BALDWIN, H. L. BERK, and L. D. PEARLSTEIN, "Turbulent Lifetimes in Mirror Machines," Phys. Rev. Lett. 36, 1051 (1976).

[22] W. C. TURNER, E. J. POWERS, and T. C. SIMONEN, "Properties of Electrostatic Ion Cyclotron Waves in a Mirror Machine," Phys. Rev. Lett. 39, 1087 (1977).

[23] J. KESNER, "ICRF Fueling of Tandem Mirror End Plugs," J. Nuclear Fusion, 19, 108 (1979).

[24] R. BREUN et al., "Experiments in a Tandem Mirror Sustained and Heated Solely by RF," Physical Review Letters 47, 1833 (1981).

[25] T. CHO et al., "Observation and Control of Transverse Energy-Transport Barrier due to the Formation of an Energetic-Electron Layer with Sheared E×B Flow," Phys. Rev. Lett. 97, 055001 (2006).

[26] V. E. MOISEENKO and O. AGREN, "Radiofrequency Heating of Sloshing Ions in a Straight Field Line Mirror," Phys. Plasma 12, 102504 (2005).

[27] C. LITWIN and N. HERSHKOWITZ, "Eigenmode electric field profiles in cylindrical plasmas," Phys. Fluids 30, 1323 (1987).

[28] J. D. HANSON and E. OTT, "An alpha-particledriven Alfven wave Instability in a Tandem Mirror reactor," Phys. Fluids 27, 150 (1984).

[29] Y. YAMAGUCHI et al., "Eigenmode Formations of $m=1$ Fast Alfvén Waves in the Ion-cyclotron Frequency Range in the GAMMA10 Central Cell," Plasma Phys. Control. Fusion 48, 1155 (2006). 